\title{
Simultaneous measurement of protein one-bond residual dipolar couplings without increased resonance overlap
}

\author{
Vinesh Vijayan, Markus Zweckstetter* \\ Department for NMR-based Structural Biology, Max Planck Institute for Biophysical Chemistry, Am Fassberg 11, 37077 Göttingen, Germany
}

Received 27 September 2004; revised 17 January 2005

Available online 12 March 2005

\begin{abstract}
A NMR strategy designed to measure simultaneously and without increased resonance overlap scalar and dipolar couplings (RDCs) in ${ }^{13} \mathrm{C}$-, ${ }^{15} \mathrm{~N}$-labeled proteins is presented. Contrary to common schemes for simultaneous measurement of RDCs, a single reference experiment is used for the extraction of more than one type of coupling, thereby reducing the required measurement time. This is accomplished by a common reference spectrum followed by a series of interleaved experiments, in which a particular coupling dependent parameter is varied according to the quantitative $J$-correlation method or using accordion spectroscopy. To illustrate this idea, we have modified the 3D TROSY-HNCO and the 3D CBCA $(\mathrm{CO}) \mathrm{NH}$ experiment allowing efficient measurement of one-bond ${ }^{1} \mathrm{D}_{\mathrm{NH}},{ }^{1} \mathrm{D}_{\mathrm{C}^{\prime} \mathrm{N}},{ }^{1} \mathrm{D}_{\mathrm{C} \alpha \mathrm{H} \alpha},{ }^{1} \mathrm{D}_{\mathrm{C} \beta \mathrm{H} \beta}$, and ${ }^{1} \mathrm{D}_{\mathrm{C} \alpha \mathrm{C}^{\prime}}$ couplings in small to medium sized proteins. In addition, the experiments are expected to be useful for largely unfolded proteins, which show strong resonance overlap but have very favorable relaxation properties. Measurement of RDCs is demonstrated on uniformly ${ }^{15} \mathrm{~N}-{ }^{13} \mathrm{C}$-labeled ubiquitin and on the sensory domain of the membraneous twocomponent fumarate sensor DcuS of Escherichia coli $(17 \mathrm{kDa})$. DcuS was found to be unstable and to precipitate in one to two weeks. RDCs obtained from these experiments are in good agreement with the $1.8 \AA \mathrm{X}$-ray structure of ubiquitin.
\end{abstract}

(C) 2005 Elsevier Inc. All rights reserved.

Keywords: Residual dipolar coupling; Unfolded protein; CBCA(CO)NH; NMR; Scalar coupling; Ubiquitin

\section{Introduction}

Residual dipolar couplings (RDCs) can be observed in solution when a molecule is aligned with the magnetic field, either as a result of its own magnetic susceptibility anisotropy [1,2] or caused by an anisotropic environment such as an oriented liquid crystalline phase [3] or an anisotropically compressed gel $[4,5]$. When alignment can be kept sufficiently weak, the NMR spectra retain the simplicity normally observed in regular isotropic solution, while allowing quantitative measurement of a wide variety of RDCs, even in macromolecules [6,7]. In proteins, the most commonly measured dipolar

\footnotetext{
* Corresponding author. Fax: +495512012202.

E-mail address: mzwecks@gwdg.de (M. Zweckstetter).
}

couplings are the one bond ${ }^{1} \mathrm{D}_{\mathrm{NH}},{ }^{1} \mathrm{D}_{\mathrm{C} \alpha \mathrm{H} \alpha},{ }^{1} \mathrm{D}_{\mathrm{C}^{\prime} \mathrm{N}}$, and ${ }^{1} \mathrm{D}_{\mathrm{C} a \mathrm{C}^{\prime}} \mathrm{RDCs}$. These couplings are sufficiently large to be measured with good accuracy, allow definition of the orientation of a peptide plane and play a key role in RDC-based structure determination [8-11].

However, it is observed that certain proteins are unstable under the orienting media condition. If the magnitude and/or the orientation of the alignment tensor are changing slowly over time due to instability of the sample (protein or liquid crystal), the different sets of dipolar couplings will not be consistent with a unique alignment tensor/structure. This will in turn bias the protein structure calculation process. Therefore, it is desirable to measure as many types of RDCs in the committed time in which the protein/liquid crystal is stable. Moreover, much focus is put on dipolar couplings for rapid structure determination, with the aim to establish 
NMR spectroscopy as an important tool in Structural Genomics [12]. Therefore, there is the need to measure a large set of dipolar couplings in an as short time as possible.

Instead of measuring one type of coupling per NMR experiment, a number of schemes have been proposed recently that allow simultaneous measurement of different types of dipolar couplings in a single spectrum: ${ }^{1} \mathrm{D}_{\mathrm{HN}} /{ }^{1} \mathrm{D}_{\mathrm{C} \alpha \mathrm{C}^{\prime}}$ or ${ }^{1} \mathrm{D}_{\mathrm{HN}} /{ }^{1} \mathrm{D}_{\mathrm{C} \alpha \mathrm{H} \alpha}$ from frequency differences using the IPAP-strategy $[13,14],{ }^{1} \mathrm{D}_{\mathrm{C} \alpha \mathrm{C}} /^{/ 3} \mathrm{D}_{\mathrm{HNC} \alpha}$ or ${ }^{1} \mathrm{D}_{\mathrm{NC \alpha}} /{ }^{2} \mathrm{D}_{\mathrm{NCa}} /{ }^{2} \mathrm{D}_{\mathrm{HNC} \alpha} /{ }^{3} \mathrm{D}_{\mathrm{HNC} \alpha}$ from $3 \mathrm{D} \operatorname{HNCO}(\alpha /$ $\left.\beta-\mathrm{C}^{\prime} \mathrm{C} \alpha-J\right)$-TROSY and $\mathrm{HNCO}(\alpha / \beta-\mathrm{NC} \alpha-J)$-TROSY [15], ${ }^{1} \mathrm{D}_{\mathrm{NC} \alpha} /{ }^{2} \mathrm{D}_{\mathrm{HNC} \alpha}$ from ${ }^{13} \mathrm{C}^{\alpha}$ coupled or $J$-modulated $\left[{ }^{15} \mathrm{~N},{ }^{1} \mathrm{H}\right]$-HSQC spectra [16], ${ }^{1} \mathrm{D}_{\mathrm{C}^{\prime} \mathrm{N}} /{ }^{1} \mathrm{D}_{\mathrm{NC} \alpha} /{ }^{2} \mathrm{D}_{\mathrm{NC} \alpha}$ from a quantitative $J$-correlation 3D TROSY-HNC experiment, in which cross and reference axial peaks are observed in a single spectrum [17], ${ }^{1} \mathrm{D}_{\mathrm{C} \alpha \mathrm{H} \alpha} /{ }^{2} \mathrm{D}_{\mathrm{NH} \alpha}$ from HNCA-TROSY [18], and ${ }^{1} \mathrm{D}_{\mathrm{C}^{\prime} \mathrm{N}} /{ }^{2} \mathrm{D}_{\mathrm{HNC}^{\prime}}$ from an E.COSY-type HSQC experiment [19]. Approaches in which multiple couplings are allowed to evolve in a single experiment have the disadvantage that each coupling results in a splitting that reduces the signal intensity by one half. Therefore, the overall sensitivity of these experiments is not higher than when the couplings are measured sequentially (potentially even lower if not multiple values for each $J+D$ value are obtained in the same experiment). In addition, if the multiplet components are not separated in different spectra this will result in increased overlap. Increased overlap is also a problem for the quantitative $J$-correlation TROSYHNC experiment, as reference peaks are located in a single plane of the $3 \mathrm{D}$ spectrum and both $\mathrm{C}^{\alpha}(i)$ and $\mathrm{C}^{\alpha}(i-1)$ peaks are present [17].

Here we present a different strategy for simultaneous measurement of dipolar couplings that is based on the quantitative $J$-correlation method and accordion spectroscopy. Only a single coupling is active during a single experiment, but for different couplings the same reference spectrum is used. This can reduce the total required measurement time without increasing resonance overlap. In addition, the experiments, in which different couplings are active, are recorded interleaved, such that measured RDCs correspond to a unique alignment tensor. To illustrate this concept, we have modified the 3D experiments TROSY-HNCO and CBCA(CO)NH for efficient measurement of ${ }^{1} \mathrm{D}_{\mathrm{NH}},{ }^{1} \mathrm{D}_{\mathrm{C}^{\prime} \mathrm{N}},{ }^{1} \mathrm{D}_{\mathrm{C} \alpha \mathrm{H} \alpha},{ }^{1} \mathrm{D}_{\mathrm{C} \beta \mathrm{H} \beta}$, and ${ }^{1} \mathrm{D}_{\mathrm{CaC}}$ couplings in small to medium sized, globular or larger, but intrinsically unstructured proteins.

\section{Description of the pulse sequences}

All presented pulse sequences can be used for measurement of one-bond isotropic scalar couplings. Dipolar couplings are obtained by performing the experiments both in an isotropic and a partially aligned phase followed by subtraction of the two measured values. The pulse sequence of the 3D TROSY-HNCO experiment for simultaneous measurement of ${ }^{1} \mathrm{D}_{\mathrm{C}^{\prime} \mathrm{N}}$ and ${ }^{1} \mathrm{D}_{\mathrm{NH}}$ couplings is shown in Fig. 1. It consists of three interleaved experiments that are based on the 3D TROSY-HNCO [20].

The backbone ${ }^{1} J_{\mathrm{C}^{\prime} \mathrm{N}}$ coupling is obtained by the quantitative $J$-correlation approach [21]. A reference experiment is recorded in which peak intensities are not strongly affected by the coupling of interest. This is achieved with the $180^{\circ}$ pulse at position (a) and with a conventional constant time ${ }^{15} \mathrm{~N}$ evolution period as depicted in panel (i). The ${ }^{15} \mathrm{~N}-\left\{{ }^{13} \mathrm{C}^{\prime}\right\}$ dephasing delay is adjusted to $2 T=66.7 \mathrm{~ms}$ and the separation of the $180^{\circ}$ pulses amounts to $\Delta=16.7 \mathrm{~ms}$. Thus, the effective dephasing time due to ${ }^{15} \mathrm{~N}-{ }^{13} \mathrm{C}^{\prime}$ couplings is $2 T-2 \Delta=33.3 \mathrm{~ms}$, i.e., close to $1 / 2^{1} J_{\mathrm{C}^{\prime} \mathrm{N}}$. To attenuate cross peak intensities according to the ${ }^{1} J_{\mathrm{C}^{\prime} \mathrm{N}}$ coupling, the $180^{\circ}$ inversion pulse on $\mathrm{C}^{\prime}$ is shifted to position (b) and the ${ }^{1} J_{\mathrm{C}^{\prime} \mathrm{N}}$ coupling is active for the entire interval. ${ }^{1} J_{\mathrm{C}^{\prime} \mathrm{N}}$ can then be calculated from the intensity ratios of the reference, $I_{\text {ref }}$, and attenuated resonances, $I_{\text {att }}$, according to

$\frac{I_{\text {att }}}{I_{\text {ref }}}=\frac{\sin \left(2 \pi^{1} J_{C^{\prime} N} T\right)}{\sin \left[2 \pi^{1} J_{C^{\prime} N}(T-\Delta)\right]}$.

This previously described approach for measurement of ${ }^{1} J_{\mathrm{C}^{\prime} \mathrm{N}}[21]$ is extended by addition of a third experiment, which is recorded in an interleaved manner with the two described above, and allows simultaneous measurement of ${ }^{1} J_{\mathrm{NH}}$ couplings. The $180^{\circ}$ inversion pulse on the carbonyls is kept at position (a), to maximize the ${ }^{15} \mathrm{~N}$ $\left\{{ }^{13} \mathrm{C}^{\prime}\right\}$ transfer amplitude. Opposite to the reference spectrum, however, an additional delay is inserted at the end of the constant time ${ }^{15} \mathrm{~N}$ evolution period that is incremented together with the ${ }^{15} \mathrm{~N}$ evolution time (Fig. 1, (ii)) [22]. During this delay, the ${ }^{15} \mathrm{~N}-\left\{{ }^{1} \mathrm{H}\right\}$ coupling evolves with respect to its directly attached proton while chemical shift evolution is refocused. Therefore, the peaks appear at $\omega \mathrm{N}-(\kappa+1) \pi^{1} J_{\mathrm{NH}}$ rather than at $\omega \mathrm{N}-\pi^{1} J_{\mathrm{NH}}$ as in the reference spectrum with ${ }^{1} J_{\mathrm{NH}}<0$. Typically $\kappa$ is set to 2 so that a particular cross peak is shifted between the spectra by ${ }^{1} J_{\mathrm{NH}} \mathrm{Hz}$ in the ${ }^{15} \mathrm{~N}$ dimension. In this way, a common reference experiment (Fig. 1; a, (i)) can be used for extraction of ${ }^{1} J_{\mathrm{C}^{\prime} \mathrm{N}}$ (Fig. 1; b, (i)) and ${ }^{1} J_{\mathrm{NH}}$ couplings (Fig. 1; a, (ii)). Note that the two $180^{\circ}$ pulses on ${ }^{1} \mathrm{H}$ and ${ }^{15} \mathrm{~N}$, which are necessary for measurement of the ${ }^{1} J_{\mathrm{NH}}$ coupling, are also present in the reference and the ${ }^{1} J_{\mathrm{C}^{\prime} \mathrm{N}}$-attenuated experiment, to have the same number and type of pulses in all experiments.

One-bond ${ }^{1} \mathrm{D}_{\mathrm{C} \alpha \mathrm{H} \alpha},{ }^{1} \mathrm{D}_{\mathrm{C} \beta \mathrm{H} \beta}$, and ${ }^{1} \mathrm{D}_{{\mathrm{C} \alpha \mathrm{C}^{\prime}}^{\prime}}$ dipolar couplings are measured with a modified version of the $3 \mathrm{D}$ $\mathrm{CBCA}(\mathrm{CO}) \mathrm{NH}$ pulse sequence, which has been enhanced by the use of adiabatic pulses [23,24]. The exper- 

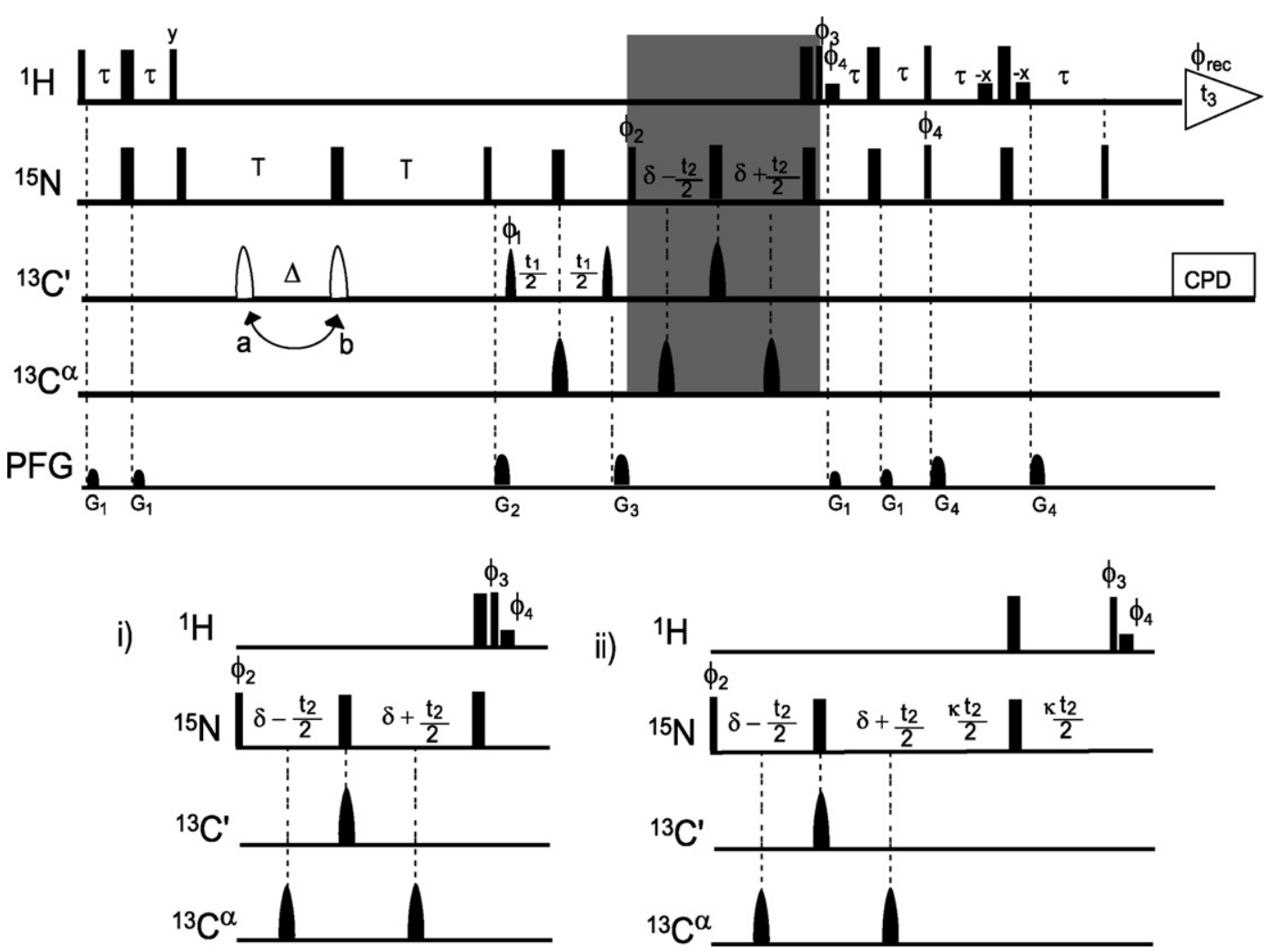

Fig. 1. Pulse scheme of the modified 3D TROSY-HNCO experiment for simultaneous measurement of ${ }^{1} J_{\mathrm{C}^{\prime} \mathrm{N}}$ and ${ }^{1} J_{\mathrm{NH}}$ couplings. Narrow and wide pulses correspond to flip angles of $90^{\circ}$ and $180^{\circ}$, respectively. All pulse phases are $x$, unless specified otherwise. ${ }^{13} \mathrm{C}^{\prime}$ pulses bracketing the $t_{1}$ evolution period are $90^{\circ}$ and have the shape of the center lobe of a $\sin x / x$ function, and durations of $120 \mu \mathrm{s}$ (at $176 \mathrm{MHz}{ }^{13} \mathrm{C}$ frequency). The other two $13 \mathrm{C}^{\prime}$ pulses are hyperbolic secant shaped $(1 \mathrm{~ms})$ and correspond to $180^{\circ}$. Composite pulse ${ }^{13} \mathrm{C}^{\prime}$ decoupling (CPD) was used during acquisition. All three $180^{\circ}{ }^{13} \mathrm{C}_{\alpha}$ pulses are sine-bell shaped and have durations of $150 \mu \mathrm{s}$. Delay durations: $\tau=2.5 \mathrm{~ms} ; \mathrm{T}=33.7 \mathrm{~ms} ; \delta=16 \mathrm{~ms} ; \Delta=16.7 \mathrm{~ms}$. For the reference and the ${ }^{1} J_{\mathrm{NH}}$-shifted spectrum, the first $180^{\circ}{ }^{13} \mathrm{C}^{\prime}$ pulse (open shape) is applied in position a; for the rephased, ${ }^{1} J_{\mathrm{C}^{\prime}} \mathrm{N}^{-}$attenuated spectrum in position b. The shaded portion of the pulse scheme is given in detail in (i) and (ii). (i) Used for the first and second interleaved spectrum and (ii) is used for the third interleaved spectrum for measurement of ${ }^{1} J_{\mathrm{NH}}$ couplings. In the shifted spectrum (ii) $\kappa$ is typically set to 2 , to obtain a shift of ${ }^{1} J_{\mathrm{NH}}$ $\mathrm{Hz}$ in the ${ }^{15} \mathrm{~N}$ dimension. Two FIDs are acquired and stored separately for obtaining quadrature selection of the TROSY component in the $t_{2}$ dimension, with phases $\phi_{2}=y, x, \phi_{3}=-y, \phi_{4}=-y$ and with phases $\phi_{2}=y,-x, \phi_{3}=y, \phi_{4}=y$ [36]. In both cases, $\phi_{1}=x, x,-x,-x$, and receiver $=x, y,-x,-y$. States-TPPI quadrature selection is used in the $\mathrm{t}_{1}$ dimension. Sine-bell shaped pulsed field gradients ( $1 \mathrm{~ms}$ each) have strength $(\mathrm{G} / \mathrm{cm})$ and axis: $G_{1}: 3, x ; G_{2}: 18, y ; \mathrm{G}_{3}: 18, x$; and $G_{4}: 18, z$.

iment is performed four times in an interleaved manner (Fig. 2). All couplings are obtained using the quantitative $J$-correlation technique. The ${ }^{1} J_{\mathrm{C} \alpha \mathrm{H} \alpha}$ and ${ }^{1} J_{\mathrm{C} \beta \mathrm{H} \beta}$ couplings are obtained from the first three interleaved experiments, in which the ${ }^{1} \mathrm{H} 180^{\circ}$ pulse during the $t_{1}$ evolution period is switched between positions $\mathrm{a}, \mathrm{b}$, and c, respectively [25]. The rephasing of the $J_{\mathrm{CH}}$ coupling of $\mathrm{C}_{\beta}$ and $\mathrm{C}_{\alpha}$ at the end of the carbon constant time period depends on the duration of $\Delta_{1}$. This is reflected in the spectra by a modulation of signal intensities according to

$\mathrm{S}\left(\Delta_{1}\right) \sim \sin \left[\pi \sum \mathrm{J}_{\mathrm{CH}}\left(2 \Delta_{1}+\Delta_{\text {eff }}\right)\right]$,

where $\Delta_{\text {eff }}$ accounts for the effect of $J_{\mathrm{CH}}$ dephasing during the adiabatic ${ }^{13} \mathrm{C}$ inversion pulse, during the ${ }^{13} \mathrm{C} 90^{\circ}$ pulses, and during short switching delays, and from which $\sum J_{\mathrm{CH}}$ can be derived by a SIMPLEX minimization routine.
${ }^{1} J_{\mathrm{CaC}^{\prime}}$ couplings are obtained from the first experiment (this is also used as a reference spectrum for the measurement of ${ }^{1} J_{\mathrm{C} \alpha \mathrm{H} \alpha}$ and ${ }^{1} J_{\mathrm{C} \beta \mathrm{H} \beta}$ couplings) and an additional fourth, interleaved experiment. To attenuate signal intensities according to the one-bond ${ }^{1} J_{\mathrm{CaC}}$ values, the $\mathrm{C}^{\prime}$ to ${ }^{15} \mathrm{~N}$ transfer step is used (Fig. 2). In all four interleaved experiments, the total dephasing delay is set to $2(\eta+\zeta)=22 \mathrm{~ms}$. In the regular $\mathrm{CBCA}(\mathrm{CO}) \mathrm{NH}$ experiment (i.e., the reference experiment) a $180^{\circ}$ inversion pulse is applied a duration $\zeta$ after the $90^{\circ}$ excitation pulse on $\mathrm{C}^{\prime}$, such that the ${ }^{1} J_{\mathrm{C}^{\mathrm{C}}}$ coupling is active for $2 \zeta=9 \mathrm{~ms}$, i.e., close to $1 / 2^{1} J_{\mathrm{CaC}}$. In the fourth interleaved experiment, however, the open $180^{\circ}$ pulse on $\mathrm{C}_{\alpha}$ is shifted by a duration $\Delta$ closer to the $180^{\circ} \mathrm{C}^{\prime}$ pulse making ${ }^{1} J_{\mathrm{CaC}^{\prime}}$ active for $2(\zeta+\Delta)=18.7 \mathrm{~ms} .{ }^{1} J_{\mathrm{C}^{\prime} \mathrm{C}^{\prime}}$ couplings are almost rephased at the end of the $\mathrm{C}^{\prime}$ to ${ }^{15} \mathrm{~N}$ transfer step, yielding weak resonance intensity. The ratio of signal intensities from the reference and attenuated spectrum follow a similar relationship as Eq. (1), 


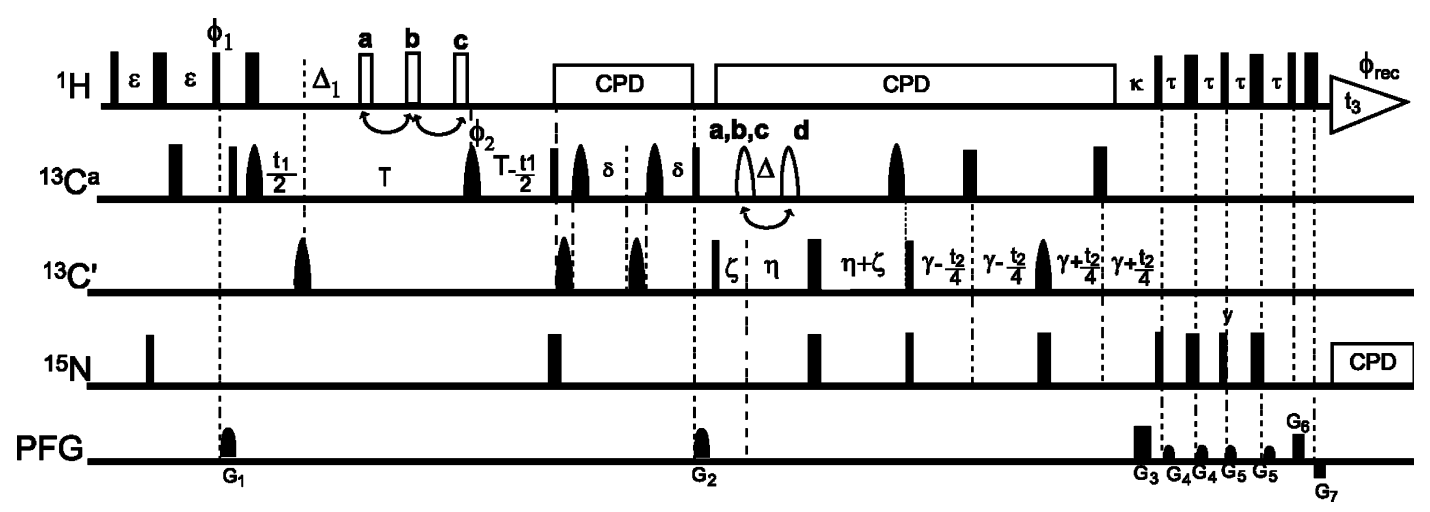

Fig. 2. Pulse scheme of the $3 \mathrm{D} \mathrm{CBCA}(\mathrm{CO}) \mathrm{NH}$ quantitative $J_{\mathrm{CH}}$ and $J_{\mathrm{Ca}^{\prime}}$ experiment. Narrow and wide pulses correspond to flip angles of $90^{\circ}$ and $180^{\circ}$, respectively. All pulse phases are $x$, unless specified otherwise. The first three ${ }^{13} \mathrm{C}^{\prime} 180^{\circ}$ pulses have a rSNOB profile [37], and a duration of $170 \mu \mathrm{s}$ (at $150 \mathrm{MHz}{ }^{13} \mathrm{C}$ frequency). All other shaped ${ }^{13} \mathrm{C}^{\prime}$ and ${ }^{13} \mathrm{C}^{\alpha / \beta}$ pulses $(46 \mathrm{ppm})$ are of the hyperbolic secant adiabatic inversion type $(500 \mu \mathrm{s})$, with a squareness level, $\mu$, of 3 [38]. The open ${ }^{1} \mathrm{H}$ pulses, applied at time point a, b, or c, are composite $\left(90^{\circ}{ }_{x}-180^{\circ}{ }_{y}-90^{\circ}{ }_{x}\right)$. Delay durations: $\varepsilon=1.3 \mathrm{~ms}$; $\Delta_{1}=0.77,1.67$, and $3.42 \mathrm{~ms}$ in the three interleaved experiments; $T=3.4 \mathrm{~ms} ; \delta=3.4 \mathrm{~ms} ; \eta=6.5 \mathrm{~ms} ; \gamma=6.8 \mathrm{~ms} ; \kappa=5.4 \mathrm{~ms} ; \tau=2.7 \mathrm{~ms}$. For the reference spectrum, the first $180^{\circ}{ }^{1} \mathrm{H}$ pulse (open shape) is applied at position a, which gives a total $J_{\mathrm{CH}}$-modulation duration of $2\left(\Delta_{1}+\Delta_{\mathrm{eff}}\right) 1.93 \mathrm{~ms}$ [25]. In the $J_{\mathrm{C} \alpha \mathrm{C}^{\prime}}$-attenuated experiment (the fourth interleaved spectrum) the open ${ }^{13} \mathrm{C}^{\alpha} 180^{\circ}$ pulse is applied at point $\mathrm{d}$, otherwise at position a/b/c. Delay durations: $\Delta=4.85 \mathrm{~ms} ; \zeta=4.5 \mathrm{~ms}$. Note that the second $180^{\circ}$ pulse on $\mathrm{C}^{\alpha}$ compensates the Block-Siegert shift, and should be applied with a reverse phase profile in order to refocus the coupling evolution during the open ${ }^{13} \mathrm{C}^{\alpha} 180^{\circ}$ pulse [39]. Phase cycling: $\phi_{1}=y,-y ;: \phi_{2}=x, x, y, y$; receiver $=x,-x,-x, x$. Rance-Kay $t_{2}$ quadrature detection is used, [40] alternating the phase of the first ${ }^{15} \mathrm{~N} 90^{\circ}$ pulse after gradient $G_{3}$ between $x$ and $-x$, in concert with the polarity of gradient $G_{3}$. States-TPPI quadrature selection is used in the $t_{1}$ dimension. Pulsed field gradients $G_{1,2,4,5}$ are sine-bell shaped ( $18 \mathrm{G} / \mathrm{cm}$ for $G_{1,2}, 28 \mathrm{G} / \mathrm{cm}$ for $\left.G_{4,5}\right)$, and $G_{3,6,7}$ are rectangular $\left(30 \mathrm{G} / \mathrm{cm}\right.$ ). Durations: $G_{1,2,3,4,5,6,7}=2,1.4,2.705,0.4,0.4,0.2,0.074 \mathrm{~ms}$, with respective gradient axes: $x y, y, z, x, y, z, z$.

from which ${ }^{1} J_{\mathrm{CaC}}$ couplings can be extracted by a one dimensional grid search or a nonlinear fit. Note that the $\mathrm{C}_{\beta}$ resonances are also modulated by the ${ }^{1} J_{\mathrm{CaC}}$ coupling. Thus, if there is signal overlap in the $\mathrm{C}_{\alpha}$ region of the spectrum one can still obtain the couplings from the $\mathrm{C}_{\beta}$ resonances, provided the particular residues do not have overlapping $C_{\beta}$ frequencies.

$3 \mathrm{D}$ CBCA $(\mathrm{CO}) \mathrm{NH}$ and TROSY-HNCO are among the most sensitive triple resonance experiments for medium sized proteins and the method is applicable to any protein which yields TROSY-HNCO and CBCA (CO)NH spectra with high $\mathrm{S} / \mathrm{N}(\geqslant 20: 1$ and $\geqslant 40: 1$, respectively) in a reasonable amount of time.

\section{Data collection}

The method is demonstrated for uniformly ${ }^{13} \mathrm{C} /{ }^{15} \mathrm{~N}$ labeled human ubiquitin (VLI Research, Philadelphia) and on the uniformly ${ }^{13} \mathrm{C} /{ }^{15} \mathrm{~N}$-labeled sensory domain of the membraneous two-component fumarate sensor DcuS of E. coli (17 kDa) [26]. One-bond couplings were measured for an isotropic sample, containing $0.8 \mathrm{mM}$ ubiquitin in $95 \% \mathrm{H}_{2} \mathrm{O}, 5 \% \mathrm{D}_{2} \mathrm{O}, 50 \mathrm{mM}$ phosphate buffer, pH 6.5 at $35^{\circ} \mathrm{C}$, or containing $0.8 \mathrm{mM}$ DcuS in $95 \%$ $\mathrm{H}_{2} \mathrm{O}, 5 \% \mathrm{D}_{2} \mathrm{O}, 50 \mathrm{mM}$ phosphate buffer, $\mathrm{pH} 6.5$ at $30{ }^{\circ} \mathrm{C}$, and a second sample containing additionally either $5 \%$ C12E5/n-hexanol $(r=0.96)$ (in case of ubiquitin) [27] or $10 \mathrm{mg} / \mathrm{ml}$ bacteriophage Pf1 (in case of DcuS) [28,29].

Spectra were recorded on BRUKER AVANCE 600 and $700 \mathrm{MHz}$ spectrometers. TROSY-HNCO experi- ments were acquired as $24^{*} \times 40^{*} \times 512^{*}$ data matrices with acquisition times of $16.8 \mathrm{~ms}\left(t_{1},{ }^{13} \mathrm{C}^{\prime}\right), 18.7 \mathrm{~ms}\left(t_{2}\right.$, $\left.{ }^{15} \mathrm{~N}\right)$, and $50 \mathrm{~ms}\left(t_{3},{ }^{1} \mathrm{H}\right)$, using 4 scans per fid and a total measuring time of $19 \mathrm{~h}$ for all three interleaved spectra. In case of the $\mathrm{CBCA}(\mathrm{CO}) \mathrm{NH}$, each interleaved spectrum was recorded as a $46^{*} \times 25^{*} \times 512^{*}$ data matrix with acquisition times of $4.9 \mathrm{~ms}\left(t_{1},{ }^{13} \mathrm{C}^{\alpha / \beta}\right), 17.5 \mathrm{~ms}$ $\left(t_{2},{ }^{15} \mathrm{~N}\right)$, and $50 \mathrm{~ms}\left(t_{3},{ }^{1} \mathrm{H}\right)$ using 8 scans per fid and a total measuring time of $48 \mathrm{~h}$ for all four interleaved spectra. Data were processed and analyzed using NMRPIPE/NMRDRAW [30].

\section{Results and discussion}

Selected regions of the spectra measured with these pulse sequences for ubiquitin in isotropic solution are shown in Figs. 3 and 4. In Fig. 3A, the first interleaved TROSY-HNCO spectrum is shown, which is used as reference for extraction of both ${ }^{1} J_{\mathrm{C}^{\prime} \mathrm{N}}$ and ${ }^{1} J_{\mathrm{NH}}$ couplings. The second interleaved spectrum is shown in Fig. 3B, in which signal intensities are modulated according to the ${ }^{1} J_{\mathrm{C}^{\prime} \mathrm{N}}$ coupling. In Fig. $3 \mathrm{C}$ resonances are not attenuated compared to Fig. 3A, but shifted in the ${ }^{15} \mathrm{~N}$ dimension by ${ }^{1} J_{\mathrm{NH}} \mathrm{Hz}$ from the reference spectrum of Fig. 3A. Fig. 4 shows selected regions of the interleaved $\mathrm{CBCA}(\mathrm{CO}) \mathrm{NH}$ experiments. In Fig. $4 \mathrm{~A}$, the reference spectrum is shown and in Figs. $4 \mathrm{~B}$ and $\mathrm{C}$ signal intensities are modulated by the ${ }^{1} J_{\mathrm{C} \alpha \omega}$ $\mathrm{H} \alpha$ and ${ }^{1} J_{\mathrm{C} \beta \mathrm{H} \beta}$ couplings. Fig. $4 \mathrm{D}$ encodes the ${ }^{1} J_{\mathrm{C} \alpha \mathrm{C}^{\prime}}$ coupling. 

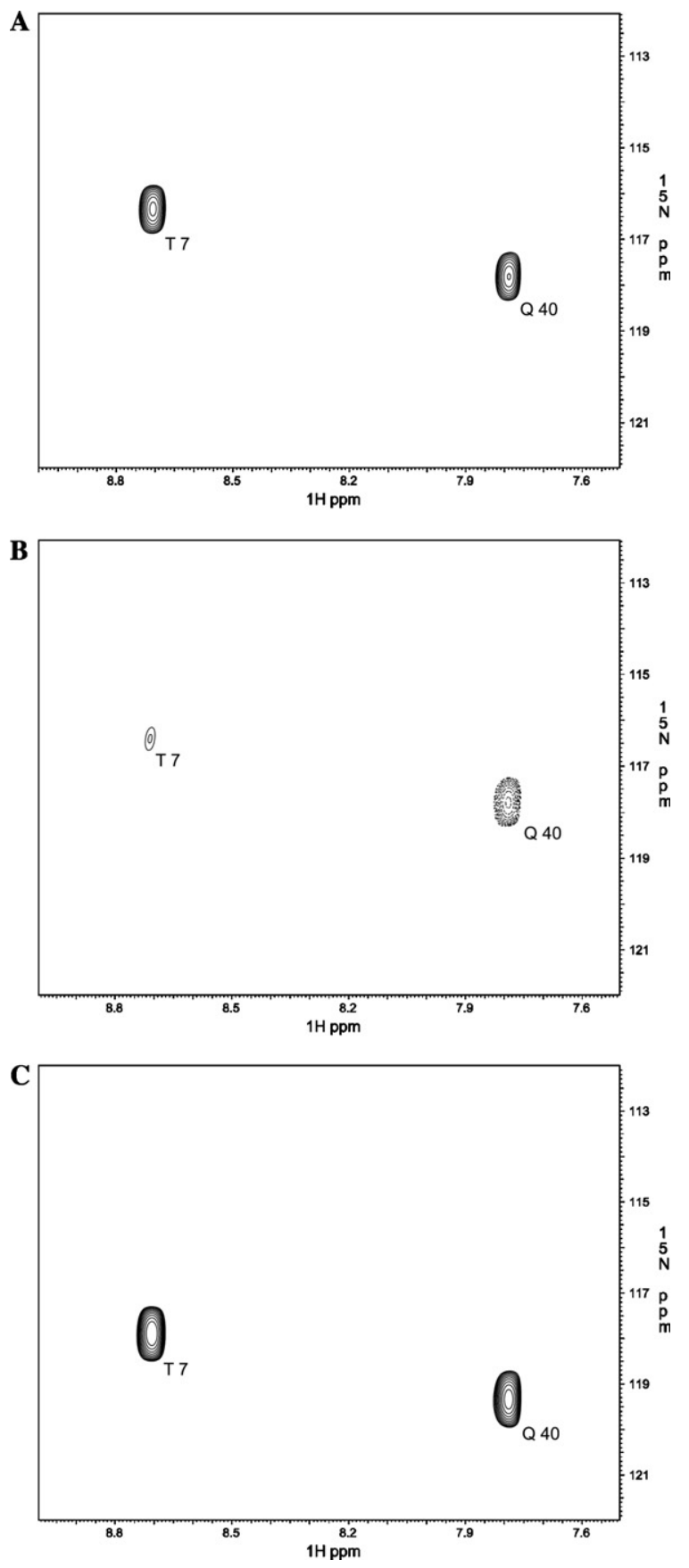

Fig. 3. Selected regions of the modified TROSY-HNCO spectrum of ubiquitin in isotropic solution. (A) reference spectrum, (B) ${ }^{1} J_{\mathrm{C}^{\prime} \mathrm{N}^{-}}$ attenuated spectrum, and (C) ${ }^{1} J_{\mathrm{NH}}$-shifted spectrum. Dashed contours denote negative intensities. Selected regions have a $C^{\prime}$ frequency of $171.1 \mathrm{ppm}$

A $\mathrm{CBCA}(\mathrm{CO}) \mathrm{NH}$ experiment is much less sensitive than a conventional HNCO due to the larger number of transfer steps and the fast relaxation of transverse $\mathrm{C}^{\alpha}$ and $\mathrm{C}^{\beta}$ magnetization. For a $11 \mathrm{kDa}$ protein such as ribonuclease $\mathrm{T} 1$ the $\mathrm{CBCA}(\mathrm{CO}) \mathrm{NH}$ is a factor of three less sensitive than a HNCO experiment (Bruker Avance 3D/Triple Resonance User Manual). For larger proteins the relative sensitivity of the $\mathrm{CBCA}(\mathrm{CO}) \mathrm{NH}$ is even further reduced. However, the following considerations have to be taken into account for measurement of ${ }^{1} \mathrm{D}_{\mathrm{C} \alpha \mathrm{C}^{\prime}}$ couplings. The sensitivity of the $\operatorname{HNCO}(\alpha / \beta$ $\mathrm{C}^{\prime} \mathrm{C}^{\alpha}-J$ ) experiment proposed by Permi et al. [15], for example, is reduced by a factor of 0.9 due to the insertion of a filter element with a duration of $9 \mathrm{~ms}$ and due to an extended ${ }^{13} \mathrm{C}$ evolution time of about $30 \mathrm{~ms}$, which is required to obtain sufficient resolution for accurate measurement of splittings (assuming a $\mathrm{C}^{\prime}$ transverse relaxation time of $117 \mathrm{~ms}$ ). Moreover, the intensity of a single resonance is split into two reducing the sensitivity by $45 \%$ overall. For the approach proposed here, on the other hand, only a single additional, conventional $\mathrm{CBCA}(\mathrm{CO}) \mathrm{NH}$ experiment is required as the reference experiment is already available either from the measurement of ${ }^{1} \mathrm{D}_{\mathrm{C} \alpha \mathrm{H} \alpha} /{ }^{1} \mathrm{D}_{\mathrm{C} \beta \mathrm{H} \beta}$ couplings or from conventional backbone assignment. No additional delays or pulses had to be introduced into the $\mathrm{CBCA}(\mathrm{CO}) \mathrm{NH}$ pulse sequence avoiding increased relaxation losses or a higher sensitivity towards pulse imperfections. The accuracy of the ${ }^{1} J_{\mathrm{CaC}^{\prime}}$ couplings obtained from the CBCA (CO) NH is further improved, as splittings can be obtained from both the $\mathrm{C}^{\alpha}$ and the $\mathrm{C}^{\beta}$ resonance. The corresponding values can be averaged, such that the sensitivity of the CBCA(CO)NH (in terms of measurement of couplings) is effectively increased by a factor of $\sqrt{ } 2$. Therefore, for small to medium sized proteins such as ribonuclease $\mathrm{T} 1$, measurement of ${ }^{1} \mathrm{D}_{\mathrm{C} \alpha \mathrm{C}^{\prime}}$ couplings simultaneously with ${ }^{1} \mathrm{D}_{\mathrm{C} \alpha \mathrm{H} \alpha} /{ }^{1} \mathrm{D}_{\mathrm{C} \beta \beta}$ values has a similar efficiency as separate measurement of ${ }^{1} \mathrm{D}_{\mathrm{C} \alpha \mathrm{C}^{\prime}}$ couplings with a $\mathrm{HNCO}\left(\alpha / \beta-\mathrm{C}^{\prime} \mathrm{C}^{\alpha}-J\right)$ experiment.

Measurement of ${ }^{1} J_{\mathrm{C} \alpha \mathrm{C}^{\prime}}$ couplings as proposed herein has the following favorable properties: (i) Opposite to $\alpha /$ $\beta$-filtered spin-state selective experiments $J$-mismatch is not a problem in quantitative $J$-correlation experiments. This is particularly important for measurement of RDCs where the filter period no longer matches the $J+D$ value. (ii) Despite the lower resolution of a $\mathrm{CBCA}(\mathrm{CO}) \mathrm{NH}$ compared a HNCO experiment, a very complete set of ${ }^{1} J_{\mathrm{C} \alpha \mathrm{C}^{\prime}}$ couplings can be obtained as in case of $\mathrm{C}^{\alpha}$ overlap the coupling might be extracted from the $\mathrm{C}^{\beta}$ resonance. (iii) For extraction of ${ }^{1} J_{\mathrm{C} \alpha \mathrm{C}^{\prime}}$ couplings no additional experiment has to be processed, peak picked and analyzed. ${ }^{1} J_{\mathrm{C} \alpha \mathrm{C}^{\prime}}$ couplings are obtained in parallel with the ${ }^{1} J_{\mathrm{C} \alpha \mathrm{H} \alpha}$ and ${ }^{1} J_{\mathrm{C} \beta \mathrm{H} \beta}$ couplings, minimizing the number of errors in extracted dipolar coupling values. (iv) The proposed pulse sequences allow simultaneous measurement of RDCs such that they will be in agreement with a unique effective alignment tensor even if the protein degrades/aggregates rapidly. With the CBCA (CO)NH this is possible for ${ }^{1} J_{\mathrm{C} \alpha \mathrm{C}^{\prime}},{ }^{1} J_{\mathrm{C} \alpha \mathrm{H} \alpha}$, and ${ }^{1} J_{\mathrm{C} \beta \mathrm{H} \beta}$ couplings without increased resonance overlap and employing a common reference experiment.

The random error in coupling values obtained by quantitative $J$-correlation methods mainly depends on the intensity in the reference spectrum, $I_{\text {ref }}$, and the ran- 

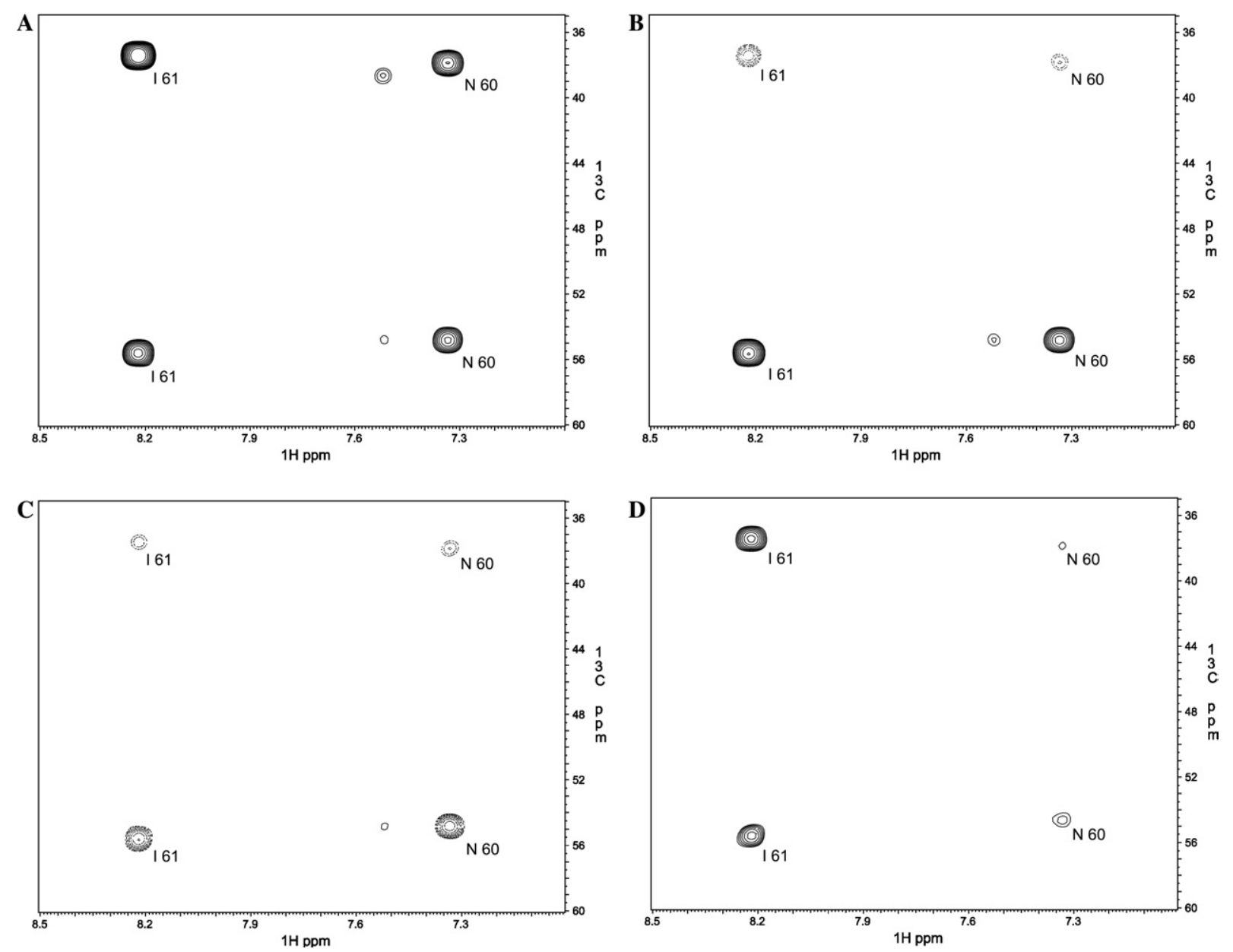

Fig. 4. Selected regions of the modified $\mathrm{CBCA}(\mathrm{CO}) \mathrm{NH}$ spectrum of ubiquitin in isotropic solution. (A) The reference spectrum. (B and $\mathrm{C}$ ) The attenuated spectra for measuring $\mathrm{C}_{\alpha}-\mathrm{H}_{\alpha}$ and $\mathrm{C}_{\beta}-\mathrm{H}_{\beta}$ couplings. (D) Spectrum for measuring $\mathrm{C}_{\alpha}-\mathrm{C}^{\prime}$ couplings. Negative intensities are denoted by dashed contours. Selected regions have a ${ }^{15} \mathrm{~N}$ frequency of $116.2 \mathrm{ppm}$.

dom noise $\sigma$ in the attenuated spectra, and can be approximated as $\sigma /\left[2 \pi(\zeta+\Delta) I_{\text {ref }}\right]$, where $2(\zeta+\Delta)$ is the total duration in which that particular coupling is active in the attenuated spectrum. So for a signal-to-noise ratio of 50 in the reference spectrum the random error in ${ }^{1} J_{\mathrm{CaC}^{\prime}}[2(\zeta+\Delta)=18 \mathrm{~ms}]$ will be $0.35 \mathrm{~Hz}$. Except for couplings between ${ }^{13} \mathrm{C}^{\prime}$ and carbons resonating in the $\mathrm{C}_{\alpha}$ region, all other couplings attenuate the reference and attenuated spectrum in the same way, and therefore do not affect the derived coupling. The inter-residue ${ }^{2} J_{\mathrm{CaC}}$ coupling, on the other hand, affect the attenuated and the reference spectrum differentially. The magnitude of ${ }^{2} J_{\mathrm{CaC}}$ with the next residue being in cis position is $\leqslant 3 \mathrm{~Hz}$. [31]. This attenuates the reference spectrum by less than $0.4 \%$ and the attenuated spectra by $1.5 \%$, and the $I_{\text {att }} / I_{\text {ref }}$ ratio is decreased by $1.2 \%$, resulting in a coupling which is at most $1.2 \%$ closer to $55 \mathrm{~Hz}$ than its real value. When the bonds are trans, as it might be the case for residues preceding proline, the coupling could be as large as $6 \mathrm{~Hz}$ [31] resulting in a more pronounced overestimation of the derived coupling. However, these residues are any way not detected in $\mathrm{CBCA}(\mathrm{CO}) \mathrm{NH}$ experiments. Due to the fact that the selectivity of the $\mathrm{C} \alpha$ inversion pulse is not perfect, other long-range couplings that may affect the derived ${ }^{1} J_{\mathrm{C} \mathrm{C}^{\prime}}$ value can include ${ }^{2} J_{\mathrm{C}^{\prime} \mathrm{C} \beta}$ and ${ }^{3} J_{\mathrm{C}^{\prime} \mathrm{C} \gamma}$. These couplings, however, are comparatively smaller. Assuming ${ }^{2} J_{\mathrm{C}^{\prime} \mathrm{C} \beta}$ and ${ }^{3} J_{\mathrm{C}^{\prime} \mathrm{C} \gamma}$ values of 3 and $4 \mathrm{~Hz}$, respectively, the cumulative effect of all the three couplings would be to make the coupling closer to $55 \mathrm{~Hz}$ by less than $4.6 \%$. So a coupling of $52.0 \mathrm{~Hz}$ will appear as $52.1 \mathrm{~Hz}$ and for an actual coupling of $54 \mathrm{~Hz}$ the derived coupling would be $54.05 \mathrm{~Hz}$, which typically is considerably smaller than the random error.

Hence, extracted coupling values do not contain any large systematic errors. This is also evident from isotropic ${ }^{1} J_{\mathrm{C} \alpha \mathrm{C}^{\prime}}$ couplings measured with the pulse sequence presented here, when compared to those obtained from a $\mathrm{C}_{\alpha}$-coupled $\mathrm{HNCO}$ experiment recorded on the third immunoglobulin-binding domain of streptococcal protein $G$ (56 residues). The coupling values from these two measurements have a correlation of 0.98 (data not shown).

Comparison of isotropic ${ }^{1} J_{\mathrm{NH}}$ couplings measured for DcuS with the 3D TROSY-HNCO experiment pre- 
sented here and those obtained from a 2D IPAP-HSQC have a correlation of 0.97 (data not shown). In case of the 3D TROSY-HNCO, the incremented delay that was introduced for the extraction of the ${ }^{1} J_{\mathrm{NH}}$ couplings (Fig. 1; a, (ii)) slightly lowers the sensitivity of the third interleaved TROSY-HNCO spectrum compared to the reference experiment. In addition, during this additional delay transverse relaxation proceeds with an effective rate that is given by the average of the individual ${ }^{15} \mathrm{~N}$ multiplet components. Therefore, for large molecules a compromise between separation of the upfield and downfield component (which increases with $\kappa$ ) and the sensitivity of this experiment has to be made. For large molecules it might be advantageous to reduce $\kappa$. At the same time, however, the $180^{\circ}$ pulses on ${ }^{15} \mathrm{~N}$ and ${ }^{13} \mathrm{C}$ could be applied simultaneously after a time $T=16 \mathrm{~ms}$, such that $2 T$ would be reduced to $32 \mathrm{~ms}$ as in a conventional TROSY-HNCO.

${ }^{1} J_{\mathrm{C}^{\prime} \mathrm{N}}$ couplings can be measured separately using a non-decoupled TROSY-HNCO, in which ${ }^{1} J_{\mathrm{C}^{\prime} \mathrm{N}}$ values are obtained from antiphase splittings in the ${ }^{15} \mathrm{~N}$ dimension [32]. To resolve the small ${ }^{1} J_{\mathrm{C}^{\prime} \mathrm{N}}$ couplings a rather long ${ }^{15} \mathrm{~N}$ acquisition time is required, which is best implemented in a semi-constant time fashion and set to a maximum value, $t_{2}^{\max }$, roughly equal to the transverse ${ }^{15} \mathrm{~N}$ relaxation time $T_{2}$ [32]. For example, for deu- terated EIN (transverse relaxation time of the downfield component at 600 and $800 \mathrm{MHz}: 118$ and $131 \mathrm{~ms}$, respectively) a ${ }^{15} \mathrm{~N}$ acquisition time of $115 \mathrm{~ms}$ was employed. Comparison of the relaxation losses in the quantitative $J$-correlation experiment, in which the ${ }^{15} \mathrm{~N}$ magnetization is $33 \mathrm{~ms}$ longer in the transverse plane than in a regular TROSY-HNCO, with those in a non-decoupled TROSY-HNCO, with a semi-constant time evolution $t_{2}^{\max }=T_{2}\left({ }^{15} \mathrm{~N}\right)$, indicates that the quantitative $J$-correlation experiment is more sensitive for $T_{2}\left({ }^{15} \mathrm{~N}\right)>100 \mathrm{~ms}$ [33]. $T_{2}\left({ }^{15} \mathrm{~N}\right)$ values larger than $100 \mathrm{~ms}$ are commonly observed at room temperature for globular proteins with a molecular weight $<16 \mathrm{kDa}$, for the downfield component of deuterated proteins up to $30 \mathrm{kDa}$, such as EIN, or for large protonated, but natively unfolded proteins. For example, for ubiquitin $\quad\left(T_{2}\left({ }^{15} \mathrm{~N}, \quad 25^{\circ} \mathrm{C}\right) \sim 160 \mathrm{~ms} ; \quad T_{2}\left({ }^{15} \mathrm{~N}\right.\right.$, $7^{\circ} \mathrm{C}$ ) $\sim 105 \mathrm{~ms}$ ) the quantitative $J$-correlation experiment is 15 and $2 \%$ more sensitive than the non-decoupled experiment at 25 and $7{ }^{\circ} \mathrm{C}$, respectively. On the other hand, for a relaxation time of $74 \mathrm{~ms}\left(\sim T_{2}\left({ }^{15} \mathrm{~N}\right)\right.$ of protonated EIN) the non-decoupled HNCO would be more sensitive by $20 \%$. Therefore, for transverse relaxation times larger than $100 \mathrm{~ms}$ simultaneous measurement of ${ }^{1} J_{\mathrm{NH}}$ and ${ }^{1} J_{\mathrm{C}^{\prime} \mathrm{N}}$ couplings as proposed here achieves a time saving that corresponds to about one
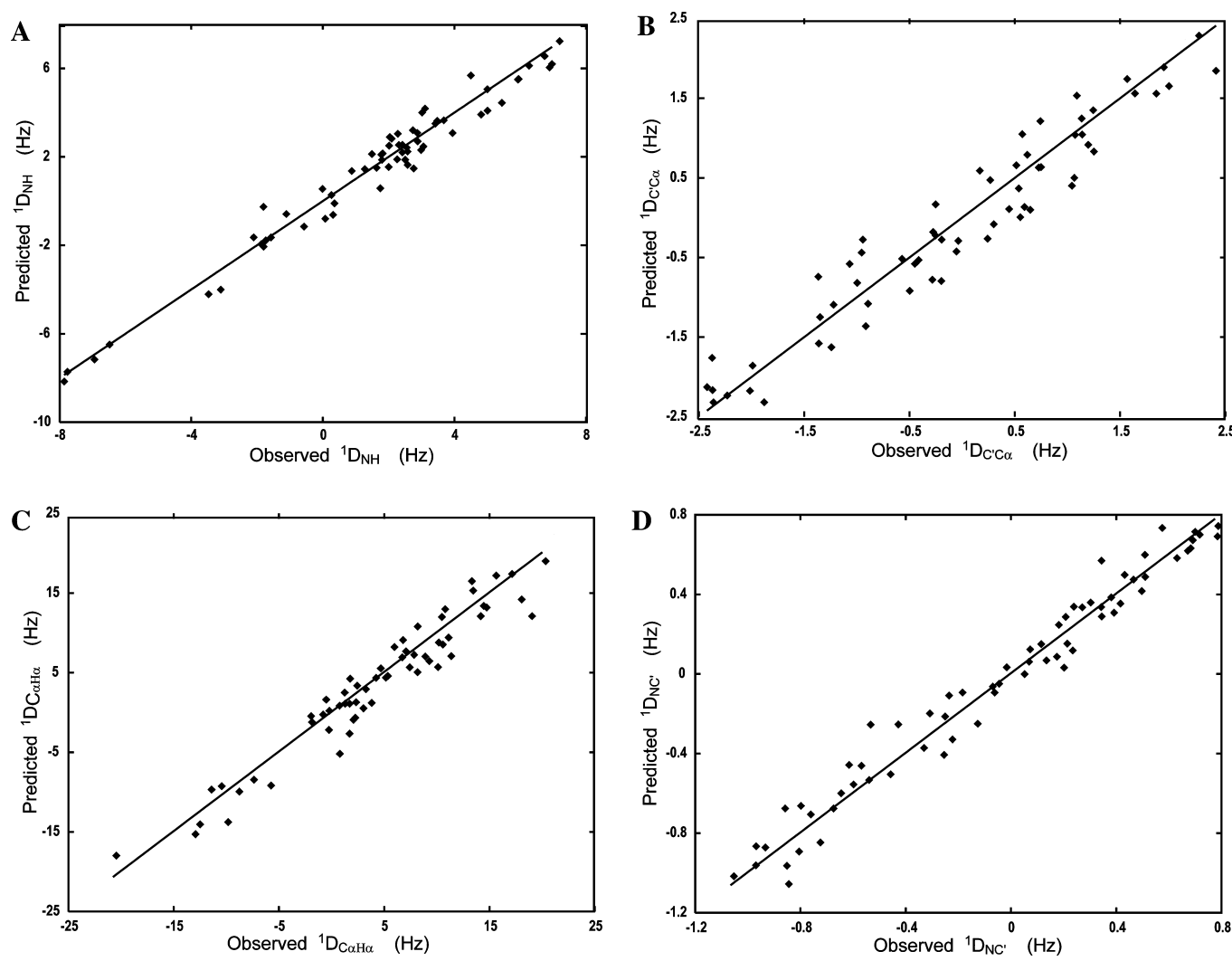

Fig. 5. Correlation between observed dipolar couplings and values back-calculated from the crystal structure of ubiquitin (PDB code: 1ubq). The correlation coefficients are $0.98,0.96,0.98$, and 0.96 for ${ }^{1} \mathrm{D}_{\mathrm{NH}},{ }^{1} \mathrm{D}_{\mathrm{C} \alpha \mathrm{H} \alpha},{ }^{1} \mathrm{D}_{\mathrm{C}^{\prime} \mathrm{N}}$, and ${ }^{1} \mathrm{D}_{\mathrm{C} \alpha \mathrm{C}^{\prime}}$ couplings, respectively. 
conventional TROSY-HNCO. Note, that this does not even take into account that the reference experiment (Fig. 1; a, (i)) or the shifted experiment (Fig. 1; a, (ii)) can be used for backbone assignment, which is difficult with the non-decoupled TROSY-HNCO. A further drawback of the non-decoupled TROSY-HNCO is that the overlap is more pronounced.

Fig. 5 compares experimental dipolar couplings with those back-calculated from the $1.8 \AA \mathrm{X}$-ray structure of ubiquitin [34] using singular-value decomposition as implemented in the program PALES [35]. Correlation factors of $0.98,0.96,0.98$, and 0.96 are obtained for ${ }^{1} \mathrm{D}_{\mathrm{NH}},{ }^{1} \mathrm{D}_{\mathrm{C} \alpha \mathrm{H} \alpha},{ }^{1} \mathrm{D}_{\mathrm{C}^{\prime} \mathrm{N}}$, and ${ }^{1} \mathrm{D}_{\mathrm{C} \alpha \mathrm{C}^{\prime}}$ couplings, respectively. This demonstrates that the pulse sequences presented here allow accurate measurement of $J$ and dipolar couplings in medium sized proteins. Residual dipolar couplings measured in DcuS (17 kDa) were used for the structure determination of this protein [26].

\section{Conclusion}

We have presented a simple strategy for simultaneous measurement of scalar and dipolar couplings in ${ }^{13} \mathrm{C}$-, ${ }^{15} \mathrm{~N}$-labeled proteins. More than one type of coupling is extracted from a single reference experiment, thereby reducing the required measurement time for small to medium sized globular, as well as larger, unfolded proteins. The couplings are obtained either using the quantitative $J$-correlation approach or accordion spectroscopy; a second spectrum is recorded in which the signal intensities are modulated or signals are shifted according to the coupling of interest. Hence, simultaneous measurement of dipolar couplings does not result in an increase in resonance overlap compared to when the couplings are measured sequentially. The strategy presented is general and can be applied to various $3 \mathrm{D}$ experiments.

The interleaved 3D TROSY-HNCO and CBCA (CO) NH experiments presented here allow measurement of four backbone RDCs and ${ }^{1} \mathrm{D}_{\mathrm{C} \beta \mathrm{H} \beta} \cdot{ }^{1} \mathrm{D}_{\mathrm{NH}},{ }^{1} \mathrm{D}_{\mathrm{C} \alpha \mathrm{H} \alpha}$, ${ }^{1} \mathrm{D}_{\mathrm{C}^{\prime} \mathrm{N}}$, and ${ }^{1} \mathrm{D}_{\mathrm{CaC}^{\prime}}$ can be used in a conventional structure calculation, enabling accurate determination of peptide plane orientations [9], or protein fold determination even in the absence of NOEs $[8,10,11]$. The combination of ${ }^{1} \mathrm{D}_{\mathrm{C} \alpha \mathrm{H} \alpha},{ }^{1} \mathrm{D}_{\mathrm{C} \beta \mathrm{H} \beta}$, and ${ }^{1} \mathrm{D}_{\mathrm{C} \alpha \mathrm{C}^{\prime}}$ provides $\chi 1$ rotamer information, even in the absence of a backbone structure [25]. Moreover, the reference spectra, which are used here for extraction of couplings, can be used for traditional backbone assignment, further reducing the total required measurement time in a NMR structure determination project. In the broader sense of speeding up structure determination by NMR (e.g., as required for Structural Genomics applications), our strategy also has the advantage that peak picking, refinement of picked peaks and reassignment of resonances only has to be done for the reference spectrum, as long as the quantitative $J$-correlation approach is employed. For extraction of the ${ }^{1} J_{\mathrm{CC}^{\prime}}$ coupling no additional experiment has to be processed, peak picked and analyzed. ${ }^{1} J_{\mathrm{C} \alpha \mathrm{C}^{\prime}}$ couplings are obtained in parallel with the ${ }^{1} J_{\mathrm{C} \alpha \mathrm{H} \alpha}$ and ${ }^{1} J_{\mathrm{C} \beta \mathrm{H} \beta}$ couplings. In addition, if the anisotropic medium is unstable or the protein degrades rapidly, as it is the case for DcuS, measured residual dipolar couplings will be in agreement with a unique effective alignment tensor.

\section{Acknowledgments}

We are grateful to Professor Unden for providing ${ }^{13} \mathrm{C} /{ }^{15} \mathrm{~N}$-labeled DcuS, to Professor Griesinger for useful discussions and to the MPG for support. M.Z. is the recipient of a DFG Emmy Noether-Fellowship (ZW71/1-4).

\section{References}

[1] A.A. Bothnerby, in: D.M. Grant, R.K. Harris (Eds.), Encyclopedia of Nuclear Magnetic Resonance, Wiley, Chichester, 1996, pp. 2932-2938.

[2] C. Gayathri, A.A. Bothnerby, P.C.M. Vanzijl, C. Maclean, Dipolar magnetic-field effects in nmr-spectra of liquids, Chem. Phys. Lett. 87 (1982) 192-196.

[3] A. Saupe, G. Englert, Phys. Rev. Lett. 11 (1963) 462-464.

[4] R. Tycko, F.J. Blanco, Y. Ishii, Alignment of biopolymers in strained gels: a new way to create detectable dipole-dipole couplings in high-resolution biomolecular NMR, J. Am. Chem. Soc. 122 (2000) 9340-9341.

[5] J. Sass, F. Cordier, A. Hoffmann, A. Cousin, J.G. Omichinski, H. Lowen, S. Grzesiek, Purple membrane induced alignment of biological macromolecules in the magnetic field, J. Am. Chem. Soc. 121 (1999) 2047-2055.

[6] N. Tjandra, A. Bax, Direct measurement of distances and angles in biomolecules by NMR in a dilute liquid crystalline medium, Science 278 (1997) 1111-1114.

[7] J.R. Tolman, J.M. Flanagan, M.A. Kennedy, J.H. Prestegard, Nuclear magnetic dipole interactions in field-oriented proteinsinformation for structure determination in solution, Proc. Natl. Acad. Sci. USA 92 (1995) 9279-9283.

[8] Y.S. Jung, M. Sharma, M. Zweckstetter, Simultaneous assignment and structure determination of protein backbones by using NMR dipolar couplings, Angew. Chem. Int. Ed. 43 (2004) 34793481 .

[9] J.J. Chou, S.P. Li, A. Bax, Study of conformational rearrangement and refinement of structural homology models by the use of heteronuclear dipolar couplings, J. Biomol. NMR 18 (2000) 217227.

[10] F. Delaglio, G. Kontaxis, A. Bax, Protein structure determination using molecular fragment replacement and NMR dipolar couplings, J. Am. Chem. Soc. 122 (2000) 2142-2143.

[11] J.C. Hus, D. Marion, M. Blackledge, Determination of protein backbone structure using only residual dipolar couplings, J. Am. Chem. Soc. 123 (2001) 1541-1542.

[12] J.H. Prestegard, H. Valafar, J. Glushka, F. Tian, Nuclear magnetic resonance in the era of structural genomics, Biochemistry 40 (2001) 8677-8685.

[13] M. Ottiger, F. Delaglio, A. Bax, Measurement of J and dipolar couplings from simplified two-dimensional NMR spectra, J. Magn. Reson. 131 (1998) 373-378. 
[14] E. de Alba, M. Suzuki, N. Tjandra, Simple multidimensional NMR experiments to obtain different types of one-bond dipolar couplings simultaneously, J. Biomol. NMR 19 (2001) 63-67.

[15] P. Permi, P.R. Rosevear, A. Annila, A set of HNCO-based experiments for measurement of residual dipolar couplings in N15, C-13, (H-2)-labeled proteins, J. Biomol. NMR 17 (2000) 4354.

[16] S. Heikkinen, P. Permi, I. Kilpelainen, Methods for the measurement of (1)J(NC alpha) and (2)J(NC alpha) from a simplified 2D C-13(alpha)-coupled N-15 SE-HSQC spectrum, J. Magn. Reson. 148 (2001) 53-60.

[17] H.L. Wienk, M.M. Martinez, G.N. Yalloway, J.M. Schmidt, C. Perez, H. Ruterjans, F. Lohr, Simultaneous measurement of protein one-bond and two-bond nitrogen-carbon coupling constants using an internally referenced quantitative J-correlated [(15)N,(1)H]-TROSY-HNC experiment, J. Biomol. NMR 25 (2003) 133-145.

[18] P. Permi, Measurement of residual dipolar couplings from $\mathrm{H}-$ 1(alpha) to C-13(alpha) and N-15 using a simple HNCA-based experiment, J. Biomol. NMR 27 (2003) 341-349.

[19] K.Y. Ding, A.M. Gronenborn, Simultaneous and accurate determination of one-bond N-15-C-13' and two-bond H-1(N)C-13' dipolar couplings, J. Am. Chem. Soc. 125 (2003) 11504 11505.

[20] M. Salzmann, K. Pervushin, G. Wider, H. Senn, K. Wuthrich, TROSY in triple-resonance experiments: New perspectives for sequential NMR assignment of large proteins, Proc. Natl. Acad. Sci. USA 95 (1998) 13585-13590.

[21] J.J. Chou, F. Delaglio, A. Bax, Measurement of one-bond N-15C-13 dipolar couplings in medium sized proteins, J. Biomol. NMR 18 (2000) 101-105.

[22] D.W. Yang, R.A. Venters, G.A. Mueller, W.Y. Choy, L.E. Kay, TROSY-based HNCO pulse sequences for the measurement of (HN)-H-1-N-15, N-15-(CO)-C-13, (HN)-H-1-(CO)-C-13, (CO)-C13-C-13(alpha) and (HN)-H-1-C-13(alpha) dipolar couplings in N-15, C-13, H-2-labeled proteins, J. Biomol. NMR 14 (1999) 333343.

[23] M. Zweckstetter, T.A. Holak, Robust refocusing of C-13 magnetization in multidimensional NMR experiments by adiabatic fast passage pulses, J. Biomol. NMR 15 (1999) 331-334.

[24] S. Grzesiek, A. Bax, Correlating backbone amide and side-chain resonances in larger proteins by multiple relayed triple resonance Nmr, J. Am. Chem. Soc. 114 (1992) 6291-6293.

[25] J.J. Chou, A. Bax, Protein side-chain rotamers from dipolar couplings in a liquid crystalline phase, J. Am. Chem. Soc. 123 (2001) 3844-3845.
[26] L. Pappalardo, I.G. Janausch, V. Vijayan, E. Zientz, J. Junker, W. Peti, M. Zweckstetter, G. Unden, C. Griesinger, The NMR structure of the sensory domain of the membranous two-component fumarate sensor (histidine protein kinase) DcuS of Escherichia coli, J. Biol. Chem. 278 (2003) 39185-39188.

[27] M. Ruckert, G. Otting, Alignment of biological macromolecules in novel nonionic liquid crystalline media for NMR experiments, J. Am. Chem. Soc. 122 (2000) 7793-7797.

[28] M.R. Hansen, L. Mueller, A. Pardi, Tunable alignment of macromolecules by filamentous phage yields dipolar coupling interactions, Nat. Struct. Biol. 5 (1998) 1065-1074.

[29] M. Zweckstetter, A. Bax, Characterization of molecular alignment in aqueous suspensions of Pf1 bacteriophage, J. Biomol. NMR 20 (2001) 365-377.

[30] F. Delaglio, S. Grzesiek, G.W. Vuister, G. Zhu, J. Pfeifer, A. Bax, Nmrpipe - a multidimensional spectral processing system based on unix pipes, J. Biomol. NMR 6 (1995) 277-293.

[31] R.H. Contreras, J.E. Peralta, Angular dependence of spin-spin coupling constants, Prog. Nucl. Magn. Reson. Spectrosc. 37 (2000) 321-425.

[32] G. Kontaxis, G.M. Clore, A. Bax, Evaluation of cross-correlation effects and measurement of one-bond couplings in proteins with short transverse relaxation times, J. Magn. Reson. 143 (2000) 184 196.

[33] M. Sattler, J. Schleucher, C. Griesinger, Heteronuclear multidimensional NMR experiments for the structure determination of proteins in solution employing pulsed field gradients, Prog. Nucl. Magn. Reson. Spectrosc. 34 (1999) 93-158.

[34] S. Vijaykumar, C.E. Bugg, W.J. Cook, Structure of ubiquitin refined at $1.8 \AA$ Resolution, J. Mol. Biol. 194 (1987) 531-544.

[35] M. Zweckstetter, A. Bax, Prediction of sterically induced alignment in a dilute liquid crystalline phase: aid to protein structure determination by NMR, J. Am. Chem. Soc. 122 (2000) 3791-3792.

[36] K.V. Pervushin, G. Wider, K. Wuthrich, Single transition-tosingle transition polarization transfer (ST2-PT) in N-15, H-1TROSY, J. Biomol. NMR 12 (1998) 345-348.

[37] E. Kupce, J. Boyd, I.D. Campbell, Short selective pulses for biochemical applications, J. Magn. Reson. B 106 (1995) 300-303.

[38] M.S. Silver, R.I. Joseph, D.I. Hoult, Highly selective Pi/2 and Pipulse generation, J. Magn. Reson. 59 (1984) 347-351.

[39] E. Kupce, R. Freeman, Compensation for spin-spin coupling effects during adiabatic pulses, J. Magn. Reson. 127 (1997) 36-48.

[40] L.E. Kay, P. Keifer, T. Saarinen, Pure absorption gradient enhanced heteronuclear single quantum correlation spectroscopy with improved sensitivity, J. Am. Chem. Soc. 114 (1992) 10663 10665 . 